\title{
Pseudomyxoma Peritonei: Diagnosis and Management
}

\author{
Khalid H. Sait ${ }^{*}$, MBCHB FRCS(C) \\ Department of Obstetrics and Gynecology, Faculty of Medicine \\ King Abdulaziz University, Jeddah, Saudi Arabia \\ khalidsait@yahoo.com
}

\begin{abstract}
Pseudomyxoma peritonei is a rare disease characterized by a large amount of mucinous ascites with peritoneal and omental implants. The etiology of the disease remains unclear. Different histological categories have been described: the benign, malignant, and the intermediate forms. It is commonly diagnosed incidentally at laparotomy. Most investigators agree that radical surgical debulking and appendectomy are the cornerstone of treatment, but the optimal management of the disease remains controversial. The role of intraoperative and intraperitoneal chemotherapy has been evaluated by a number of authors. The clinical outcomes vary widely between the different histological types and treatment modalities.
\end{abstract}

Keywords: Pseudonyxoma peritonei, Intraperitoneal chemotherapy.

\section{Introduction}

Pseudomyxoma peritonei (PMP) is a poorly understood disease. It is characterized by the presence of massive amounts of mucinous ascites with peritoneal and omental implants, that over time fill the peritoneal cavity. It was first described by Werth in 1884 as massive amounts of mucinous ascites found in association with benign ovarian neoplasm ${ }^{[1]}$.

There is considerable debate regarding the appropriate classification and type of tumor to be included in this syndrome. The main controversy relates to the inclusion or exclusion of the more "malignant' forms of the disease and to the

*To whom all correspondence \& reprint requests: P.O. Box 80215, Jeddah 21589 Saudi Arabia. Accepted for publication: 03 November 2004. Received: 15 March 2004. 
site of origin of the tumor ${ }^{[2]}$ with advances in immunocytochemistry and genetic analysis, progress has been made in more clearly categorizing this entity.

\section{Incidence}

PMP is a rare disease that is more common in women than men. Seventy-five percent of patients with PMP are female. The median age is 53-years-old (range 33- to 79-years-old). The estimated incidence is 2 of every 10,000 laparotomies performed ${ }^{[3]}$.

\section{Pathogenesis}

The pathogenesis of PMP is obscure. Werth postulated that the peritoneal surfaces undergo a peculiar reaction in response to the jelly-like material released from a cyst when it ruptures ${ }^{[1]}$. Osborn hypothesized that histological benign mucinous epithelium released by the rupture of benign cysts implants on the peritoneal surfaces and proliferates, causing $\mathrm{PMP}^{[4]}$. They postulated the existence of a tumorigenic stimulus causing multifocal neoplasms or metaplasia of the peritoneal surfaces. The historic experiments by Shanks seemed to support the idea that PMP is caused by some type of irritating agent or tumorigenic stimulus ${ }^{[1]}$. In addition the presence of mucinous pathology in both the ovary and appendix, a common finding at the time of initial laparotomy for PMP, is consistent with widespread mucinous change in the peritoneal cavity.

\section{Histology}

Histological diagnosis describes two main diagnostic categories: the benign form-disseminated peritoneal adenomucinosis (DPAM), and a malignant formperitoneal mucinous carcinomatosis (PMCA) ${ }^{[2]}$. A third category has also been described; an intermediate form which is called peritoneal mucinous carcinomatosis with intermediate or discordant features (PMCA-I/D). It has cytological features of both DPAM and PMCA. This classification was first proposed by Ronnett et al. and is important as it has prognostic significance ${ }^{[2,5,6]}$. As expected patients with the peritoneal adenomucinosis form have a significantly more favorable prognosis than patients with peritoneal mucinous carcinomatosis. Histological features of DPAM include peritoneal lesions composed of abundant extracellular mucin containing scant simple to focally proliferate mucinous epithelium with little cytologic atypia or mitotic activity; it may or may not be associated with an appendiceal mucinous adenoma ${ }^{[2]}$. Cases classified as PMCA are characterized by peritoneal lesions composed of more abundant mucinous epithelium with the architectural and cytologic features of carcinoma ${ }^{[2]}$. In some cases an associated mucinous adenocarcinoma may be 
found. In a clinicopathologic analysis of 109 cases, about 60 percent were classified as DPAM-consistent with an origin from an appendiceal mucinous adenoma. About 28 percent were classified as PMCA-consistent with origin from an appendiceal or intestinal mucinous adenocarcinoma. Approximately 13 percent of the cases with intermediate features were derived from well-differentiated appendiceal or intestinal mucinous adenocarcinomas ${ }^{[2]}$.

\section{Origin}

There is continued debate regarding the site of origin of PMP. It is most commonly associated with mucinous tumors of the appendix or the ovary. Women with PMP may have both, appendiceal and ovarian mucinous tumors. The ovarian tumors in most of these cases are secondary to appendiceal tumors ${ }^{[7-9]}$, but it may be difficult to determine the histogenetic origin of these tumors. PMP has been reported rarely in association with mucinous carcinomas of other organs, including the bile ducts, stomach, pancreas colon, fallopian tube, uterine corpus, urachus, urinary bladder, breast, and lung ${ }^{[3]}$. Despite the increasing research regarding the origin of PMP using flow cytometry, genetic analysis, and immunopathologic analysis, conflicting results are still reported. Over all it seems the vast majority of PMP cases have been shown to result from primary mucinous adenomas of the appendix ${ }^{[7,8]}$.

\section{Clinical Presentation}

PMP is commonly diagnosed incidentally at laparotomy, which is usually performed for other indications such as suspected appendicitis or ovarian carcinoma. There is broad spectrum of clinical presentations which reflects the diffuse nature of the disease within the peritoneal cavity as well as the wide range of tumor histopathological features. Patients with PMP usually have marked abdominal distension - out of proportion to the sign and symptoms of abdominal malignancies. Abdominal or palpable ovarian masses and hernias are other clinical manifestations are commonly seen. Nausea, vomiting, fatigue, and urinary tract symptoms have also been reported ${ }^{[10]}$. The range of the presenting symptoms is wide and dependant on the anatomic site of the disease. Abdominal pain, weight loss, and gastrointestinal malfunction occur relatively late in the course of this disease. The bowel eventually becomes so encased in mucin and mucinous globules that it can not function, and ultimately patients die of bowel obstruction. Rare presenting problems include cholecystitis, infertility investigation, postmenopausal bleeding, abnormal Papanicolaou smear, pelvic pain, pelvic masses, surgery for fibrosis, laparoscopy for tubal ligation, abdominal aortic aneurysm repair, deep vein thrombosis, rectal bleeding, nephrectomy, and anemia ${ }^{[11]}$. A case of intestinal obstruction has also 
been reported ${ }^{[12]}$. A rare presentation of an inguinal hernia with splenic metastasis was reported ${ }^{[13]}$. Extra-peritoneal spread of PMP is infrequent. There are few reports in the medical literature of pleural extension of mucinous tumor in patients with PMP syndrome; these are associated with a poor prognosis ${ }^{[14]}$. Pulmonary parenchymal metastasis, treated with thoracotomy and excision of metastatic nodules ${ }^{[15]}$.

\section{Diagnosis}

The diagnosis of PMP is often difficult; it usually has an insidious presentation. Ultrasonography is frequently performed as the initial diagnostic procedure. Typical findings are non-mobile, echogenic ascites with multiple semisolid masses and scalloping of the hepatic and splenic margins ${ }^{[16]}$. Furthermore, an ultrasound may be helpful in diagnostic and therapeutic paracentesis, as mucin is difficult to aspirate.

Computer tomography (CT) of the abdomen and pelvis is the most widely applied technology and has been used with great success in the diagnosis of the PMP syndrome. CT findings are often highly suggestive of PMP and sometimes these are pathognomonic ${ }^{[17]}$. The most common finding is a large volume of mucinous ascites, which has the density of fat, and displaces the small bowel and the normal mesenteric fat. Other characteristic findings are omental thickenings, multiseptate lesions, scalloping of organs, and curvilinear calcifications $^{[16-18]}$.

The role of magnetic resonance imaging (MRI) in the diagnosis of PMP is not clear. There is no data to suggest that MRI is better than CT. It has been reported that MRI may be particularly sensitive in detecting visceral invasion, but this is based on a single study of three patients ${ }^{[18]}$. At this stage, this mode remains investigational. Sugarbaker et al. have used CT as a tool for differentiating between peritoneal adenomatosis and peritoneal carcinomatosis ${ }^{[19]}$. Plain radiography and contrast studies are not particularly useful in the diagnosis of PMP. Sometimes large mucinous abdominal masses may present with displacement of the bowel centrally or with obliteration of the border of the psoas muscle ${ }^{[20]}$; rarely, punctuate or curvilinear calcifications may be noted. Double contrast techniques may demonstrate the lack of bowel lumen involvement that is characteristic of the non-invasive deposits of $\mathrm{PMP}^{[3]}$. The gross appearance is characteristic. The abdomen is filling with several liters of thick gelatinous mucoid material and the bowel is encased with mucin. Mucin globules are found within the omentum, studding the peritoneal surfaces, and floating throughout the cavity. Perhaps, the most consistent observation during surgical exploration of the patient with PMP is the complete or nearly complete 
absence of mucinous tumor on intestinal surfaces with the exception in the antrum of the stomach and pylorus, the ileocecal valve region, and the rectosigmoid colon within pelvis. All three of these sites are fixed to the retroperitoinum which are not actively and freely moving as a result of peristaltic activity. Apparently, the continuous peristaltic activity of the small bowel prevents mucinous tumor implantation on these surfaces. To a lesser extent, peristalsis inhibits the adenomatous epithelial cells from settling onto the peritoneal surfaces of the stomach and colon. Loculate mucinous cysts are present in the ovaries and/or appendix. Histological diagnosis is required and remains the "golden standard". This can be achieved by paracentesis, laparoscopy, or laparotomy.

\section{Treatment}

There is currently no accepted standard treatment for PMP. The treatment of PMP traditionally involved repeated operative procedures over several years until there were no further surgical options. Management options varied considerably and often reflected the extent of disease, histological subtype, and general conditions of the patient and surgical preference of the treating doctor options included: observation, surgery, laparoscopic intervention, chemotherapy, radiotherapy, immunotherapy, and mucolytic agents. All of these were utilized in the management of the disease.

\section{Surgery}

Surgical debulking and appendectomy are widely regarded as the mainstay of treatment of PMP syndrome. In treatment of the PMP, limited debulking and evacuation of mucinous ascites provide for short-term palliation. This is in contrast to a high likelihood of prolonged remission or cure with complete cytoreduction and perioperative intraperitoneal chemotherapy ${ }^{[21]}$.

\section{Sugarbaker Technique}

The combination of intra-abdominal 5-Fluorouracil (5-FU) and cytoreduction surgery was reported by Sugarbaker ${ }^{[21]}$ from the Washington Cancer Institute, Washington Hospital Center, in Washington, D.C. for patients with extensive mucinous tumor of gastrointestinal origin. Because of the limited penetration of tissue with intraperitoneal chemotherapy, result is largely dependent on the volume of residual tumor; a series of peritoneotomy procedures were developed. These involved stripping the partial peritoneum and ressecting structure at fixed sites that contain visceral peritoneum to accomplish a 
complete cytoreduction. Essential to the performance of peritoneotomy procedures is dissection and tumor evaporation by laser mode or electrosurgery using a ball - Tip electrosurgical hand piece in a cutting mode (Table 1). With this extensive cytoreductive approach combined with early intraperitoneal chemotherapy, the mortality rate was $2.7 \%$. The main morbidity included pancreatitis $(7.1 \%)$, fistula formation $(4.7 \%)$, and anastomotic leaks were similar to general surgical techniques at $2.4 \%$. Although, these data are impressive, there was no control group. It is unlikely that randomized trials in this setting will be performed.

Table 1. Full cytoreduction in patients with Pseudomyxoma peritonei (Sugarbaker) ${ }^{[21]}$.

\begin{tabular}{|l|}
\hline Greater omentectomy \\
\hline Lesser omentectomy \\
\hline Splenectomy \\
\hline Stripping of left diaphragm \\
\hline Stripping of right diaphragm \\
\hline Cholecystectomy \\
\hline Antrectomy \\
\hline Pelvic Peritoneotomy \\
\hline Ressection of rectosigmoid colon \\
\hline Right hemicolectomy \\
\hline
\end{tabular}

\section{Chemotherapy}

The role of chemotherapy in PMP is yet to be defined. There are insufficient data regarding the role of adjuvant chemotherapy. PMP is an ideal tumor to be considered for intensive regional chemotherapy. It is an intraperitoneal tumor with no lymphatic or hematogenous spread.

There are no randomized data evaluating the role of adjuvant chemotherapy. Results from phase II trials and retrospective reviews were conflicting. The most widely used chemotherapeutic agents are 5-FU, cyclophosphamide, mitomycin C, and cisplatinum. The Mayo Clinic series ${ }^{[22]}$ retrospective non-randomized analysis showed that survival rates were significantly better in patients treated with intraperitoneal infusions than in those treated with systemic chemotherapy. Sugarbaker et al. have described a protocol of intra-operative intraperitoneal chemotherapy with mitomycin $\mathrm{C}$, heated to $44^{\circ} \mathrm{C}$, that was initiated after complete resection of the tumor, followed by intraperitoneal 5-FU for 5 days. They reported a 5-year survival rate of approximately $90 \%$ in the most favorable prognostic group of patients ${ }^{[23]}$. 


\section{Intraperitoneal Chemotherapy}

The first attempt to use intraperitoneal chemotherapy in PMP patients was reported in 1969 by Long et al. ${ }^{[24]}$. The extremely high and sustained levels of cytotoxic chemotherapeutic agents can be achieved in the abdomen the rationale for intraperitoneal therapy is that:

1. Direct infusion of the drugs into the peritoneal cavity permits delivery of high concentrations of drug to abdominal and pelvic surfaces where the tumor is located without producing toxic systemic levels.

2. The water-soluble mucus can not metabolize chemotherapeutic agent in the peritoneal cavity.

3. PMP superficially invades intra abdominal organs and rarely metastasis, allowing maximum exposure to the drug for long period.

A number of intraperitoneum chemotherapy regimens have been used, but it is extremely difficult to evaluate the results of these, because of not only the small numbers of patient in varies series, but also the protracted length of the natural history of this condition. A major problem in the past with intraperitoneal chemotherapy delivery involved a non-uniform drug distribution, resulting from intestinal adhesion from tissue surfaced closed off by sutures, and from pooling of intraperitoneal fluid at dependent sites. Another problem with intra-abdominal drug, delivery involved the minimal penetration of tissue by chemotherapy. Chemotherapy enters tumor nodules by simple diffusion and penetrated only a few cell layers. The use of chemotherapy in the operating room after complete dissection of an adhesive process and before bowel anastomosis was completed and with the abdominal incision widely open has been described ${ }^{[25]}$. These innovative strategies for the use of intraoperative chemotherapy were reported first by the Japanese and then further developed in United States ${ }^{[23]}$. Chemotherapy treatment in the operating room has been well tolerated. If it is used, the addition of intraperitoneal hyperthermia can be used. Heat alone has an anticancer effect and it also synergizes the cytotoxicity of chemotherapy; in addition, the penetration of drug into tissue is augmented by heat. During the hyperthermal perfusion, careful monitoring of body temperature and intra-abdominal temperature must be maintained. Temperature at the infusion is approximately $44^{\circ} \mathrm{C}$ and the intra-abdominal temperature is approximately $41^{\circ} \mathrm{C}$. The core temperature remains below $39.5^{\circ} \mathrm{C}$. Heated intraoperative intraperitoneum chemotherapy is limited to certain institutions where facilities are available using this technique to meet the standard for the patient care and staff safety.

In patients with PMP, immediate postoperative intraperitoneal chemotherapy with 5-FU is used. The standardized orders for early postoperative intraperitoneal 5-FU are followed for postoperative days 1 through 5 . In the 6 
hours after the intraperitoneal 5-FU installation, the patient's bed remains flat or tilted with the head slightly down. The patient's position is changed every half-hour from the extreme right- to the extreme left-side to promote maximal gravity distribution of intraperitoneal chemotherapy. The use of chemotherapy after complete removal of cancer maximizes the complete eradication of the malignancy because only minute foci of disease need treatment. Further evaluations of intraperitoneum chemotherapy with 5-FU were conducted at National Cancer Institute in Bethesda, Maryland, USA.

\section{Other Treatment Modalities}

Green et al., first used a dextrose solution to "loosen" the peritoneal mucinous deposits ${ }^{[26]}$. The authors found that intraoperative irrigation or percutaneous lavage with dextrose and water will expedite the removal of mucus and prevent re-accumulation, although, the exact mechanism for the latter has not been determined. The use of $10 \%$ dextrose has been reported to be associated with hyperglycemia.

Intraperitoneal radioisotopes and external radiotherapy have also been used, but experience with these agents is limited ${ }^{[27]}$. Fukuma et al. have described anecdotal cases of successful treatment of PMP by immunotherapy with a streptococcal preparation, OK-432, as adjuvant treatment after surgery ${ }^{[28]}$.

\section{Prognosis}

With such a wide spectrum of disease ranging from a benign to a malignant form and wide variations in treatment modalities, it is not surprising that prognosis has been difficult to accurately define. This is further complicated by the fact that the estimation of recurrence rates was based on different series with small numbers of patients and different inclusion criteria and different therapeutic modalities.

A number of prognostic features have been identified by Sugarbaker et al. ${ }^{[23]}$. These are histopathologic types with the extent of cytoreduction, and previous surgical interventions. They reviewed the prognostic factors for 120 patients with PMP of colonic or appendix primary with low grade of tumor biology where an attempt made to clear the abdomen by tumor resection and peritoneotomy procedures.

The patients were treated with repeated abdominal lavage immediately after surgery was completed. Also, all patients were treated post operatively with intraperitoneal chemotherapy. 46 patients out of 120 patients failed treatment, even though no distance disease was present. Of these 46 patients, 37 (80\%) had persisting disease on the peritoneal surfaces. In 10 patients who had 
adequate aggressive cytoreduction procedure and in whom the disease recurred at a later time were considered to have medical treatment failure.

Patients with complete cytoreduction and adenomucinosis had a 5-year survival rate of $86 \%$, and patients with intermediate histological features had a 5 -year survival rate of 50\%. Patients with incomplete cytoreduction had 5-year survival rate of $20 \%$ and $0 \%$ at 10 years. These authors concluded that improvements in the cytoreductive approach await the development of surgically technologies to increase the total clearance of cancer from the abdominal cavity and chemotherapy treatment that is complete enough to sustain control of small-volume residual disease on all peritoneal surfaces ${ }^{[21]}$.

Gough et al. ${ }^{[27]}$ at the Mayo Clinic reported a large number of patients (65 patients) with PMP in single institution over a 26-years-interval. Minimal debulking surgeries were performed in a majority of the patients. $10 \%$ had optimal cytoreduction surgery. Thirteen percent $(13 \%)$ of the patients were given systemic chemotherapy, in $27 \%$ intrapertonium chemotherapy was given and $18 \%$ received intrapertonium radioisotope, $28 \%$ receive radiation treatment. No patients received any mucolytic agent. Median follow-up was 12-years (925.6 -years). $76 \%$ of patients had recurrence, the majority of whom treated with reoperation followed by adjuvant therapy. The median survival was 5-9 years (range 0.3-25.6 years).

The estimated 1-, 2-, 5- and 10-years survival rates were 98\%, 86\%, 53\%, and $32 \%$, respectively. They found that systemic chemotherapy associated with shorter survival rate and they concluded that aggressive surgical debulking followed by intraperitoneal radioisotope or chemotherapy should be considered in patients with $\mathrm{PMP}^{[27]}$. Wertheim et al. reported 23 cases of PMP with median follow-up 2.5 years (range 3 months to 31-years-old) in which all patients underwent surgical minimal cytoreduction. Eleven patients received post operative therapy -9 recurred. Twelve patients received no therapy - only 3 recurred. They concluded that adjuvant therapy has not been shown to be beneficial $^{[29]}$.

\section{Recurrence}

PMP is associated with high recurrence rate. According to the data from the Mayo Clinic series based on their study of 56 cases of PMP, $76 \%$ of patients developed recurrence and $50 \%$ of recurrences occurred within 2.5 years ${ }^{[22]}$. Most of the patients had carcinoma of the appendix (52\%) or ovary $(34 \%)$. According to their data, 5- and 10-year survival rates were 53\% and $32 \%$, respectively, and the median survival was 5.9 years. Another study by Witkamp et al., report a 5-year survival of $27 \%$ for peritoneal carcinomatosis of 
colorectal origin and of $86 \%$ for cases of PMP after complete cytoreduction and intraperitoneal chemohyperthermia ${ }^{[30]}$.

\section{Conclusions}

PMP is a rare and complex disease with a broad spectrum of presentation and outcomes. Definitive data regarding the optimal approach to managing this disease are not available due to the paucity of randomized trials. Unfortunately, it is unlikely that these trials will be performed due to the rarity of this disease. This highlights the importance of developing groups dealing with these rare tumors to allow formal data collection and to provide some guidance as to optimal therapeutic options in managing these patients.

\section{References}

[1] Shanks HGI. Pseudomyxoma peritonei. J Obstet Gynecol Br Common W 1961; 68: 212-224.

[2] Ronnett BM, Zahn CM, Kurman RJ, Kass ME, Sugarbaker PH, Shmookler BM. Disseminated peritoneal adenomucinosis and peritoneal mucinous carcinomatosis. A clinicopathologic analysis of 109 cases with emphasis on distinguishing pathologic features, site of origin, prognosis, and relationship to "pseudomyxoma peritonei". Am J Surg Pathol 1995; 19(12): 1390-1408.

[3] Sherer DM, Abulafia O, Eliakim R. Pseudomyxoma peritonei: a review of current literature. Gynecol Obstet Invest 2001; 51(2): 73-80.

[4] Osborn LC. Pseudomyxoma peritonei. Report of seven cases. Gynecol Oncol 1973; 1: 195-202.

[5] Yan H, Pestieau SR, Shmookler BM, Sugarbaker PH. Histopathologic analysis in 46 patients with pseudomyxoma peritonei syndrome: failure versus success with a second-look operation. Mod Pathol 2001; 14(3): 164-171.

[6] Jackson SL, Fleming RA, Loggie BW, Geisinger KR. Gelatinous ascites: a cytohistologic study of pseudomyxoma peritonei in 67 patients. Mod Pathol 2001; 14(7): 664-671.

[7] Young RH, Gilks CB, Scully RE. Mucinous tumors of the appendix associated with mucinous tumors of the ovary and pseudomyxmona peritonei. A clinicopathological analysis of 22 cases with supporting an origin in the appendix. Am J Surg Pathol 1991; 15 (5): 415-29.

[8] Prayson RA, Hart WR, Petras RE. Pseudomyxoma peritonei. A clinicopathologic study of 19 cases with emphasis on site of origin and nature of associated ovarian tumors. $\mathrm{Am} \mathrm{J}$ Surg Pathol 1994; 18(6): 591-603.

[9] Ronnett BM, Kurman RJ, Zahn CM, Shmookler BM, Jablonski KA, Kass ME, Sugarbaker PH. Pseudomyxoma peritonei in women: a clinicopathologic analysis of 30 cases with emphasis on site of origin, prognosis, and relationship to ovarian mucinous tumors of low malignant potential. Hum Pathol 1995; 26(5): 509-524.

[10] Hinson FL, Ambrose NS. Pseudomyxoma peritonei. Br J Surg 1998; 85(10): 1332-1339.

[11] Esquivel J, Sugarbaker PH. Clinical presentation of the Pseudomyxoma peritonei syndrome. Br J Surg 2000; (10): 1414-1418.

[12] Nawaz A, Karakurum A, Weltman D. Pseudomyxoma peritonei manifesting as intestinal obstruction. South Med J 2000; 93(9): 891-893. 
[13] Shimoyama S, Kuramoto S, Kawahara M, Yamasaki K, Endo H, Murakami T, Kaminishi M. A rare case of pseudomyxoma peritonei presenting an unusual inguinal hernia and splenic metastasis. J Gastroenterol Hepatol 2001; 16(7): 825-829.

[14] Pestieau SR, Esquivel J, Sugarbaker PH. Pleural extension of mucinous tumor in patients with pseudomyxoma peritonei syndrome. Ann Surg Oncol 2000; 7(3): 199-203.

[15] Mortman KD, Sugarbaker PA, Shmookler BM, DeGuzman VC, Soberman MS. Pulmonary metastases in pseudomyxoma peritonei syndrome. Ann Thorac Surg 1997 64(5): 434-436.

[16] Seshul MB, Coulam CM. Pseudomyxoma peritonei: computed tomography and sonography. AJR Am J Roentgenol 1981; 136(4): 803-806.

[17] Matsumi H, Kozuma S, Osuga Y, Yano T, Yoshikawa H, Tsutsumi O, Taketani Y. Ultrasound imaging of pseudomyxoma peritonei with numerous vesicles in ascitic fluid. Ultrasound Obstet Gynecol 1999; 13(5): 378-379.

[18] Low RN, Barone RM, Lacey C, Sigeti JS, Alzate GD, Sebrechts CP. Peritoneal tumor: MR imaging with dilute oral barium and intravenous gadolinium-containing contrast agents compared with unenhanced MR imaging and CT. Radiology 1997; 204(2): 513-520.

[19] Sugarbaker PH, Ronnett BM, Archer A, Averbach AM, Bland R, Chang D, Dalton RR, Ettinghausen SE, Jacquet P, Jelinek J, Koslowe P, Kurman RJ, Shmookler B, Stephens AD, Steves MA, Stuart OA, White S, Zahn CM, Zoetmulder FA. Pseudomyxoma peritonei syndrome. Adv Surg 1996; 30: 233-280.

[20] Walenskey RP, Venbrux AC, Prescott CA, Osterman FA Jr. Pseudomyxoma peritonei. AJR Am J Roentgenol 1996; 167(2): 471-474.

[21] Sugarbaker PH. Cytoreductive surgery and peri-operative intraperitoneal chemotherapy as a curative approach to pseudomyxoma peritonei syndrome. Eur J Surg Oncol 2001; 27(3): 239-243.

[22] Smith JW, Kemeny N, Caldwell C, Banner P, Sigurdson E, Huvos A. Pseudomyxoma peritonei of appendiceal origin. The Memorial Sloan-Kettering Cancer Center experience. Cancer 1992; 70(2): 396-401.

[23] Sugarbaker PH, Cunliffe W, Belliveau, de Bruijn E, Graves T. Rational for perioperative intraperitoneal chemotherapy as surgical adjuvant for gastrointestinal malignancy. Reg Cancer Treat 1988; 1: 66-79.

[24] Long RT, Spratt JS Jr, Dowling E. Pseudomyxoma peritonei. New concepts in the management with a report of seventeen patients. Am J Surg 1969; 117(2): 162-169.

[25] Elias DM, Ouellet JF. Intraperitoneal chemohyperthermia: rationale, technique, indications, and results. Surg Oncol Clin N Am 2001; 10(4): 915-933, xi.

[26] Green N, Gancedo H, Smith R, Bernett G. Pseudomyxoma peritonei-nonoperative management and biochemical findings. A case report. Cancer 1975; 36(5): 1834-1837.

[27] Gough DB, Donohue JH, Schutt AJ, Gonchoroff N, Goellner JR, Wilson TO, Naessens JM, O'Brien PC, van Heerden JA. Pseudomyxoma pertonei. Long-term patient survival with an aggressive regional approach. Ann Surg 1994; 219(2): 112-119.

[28] Fukuma K, Matsuura K, Shibata S, Nakahara K, Fujisaki S, Maeyama M. Pseudomyxoma peritonei: effect of chronic continuous immunotherapy with a streptococcal preparation, OK-432 after surgery. Acta Obstet Gynecol Scand 1986; 65(2): 133-137.

[29] Wertheim I, Fleischhacher D, McLachlin CM, Rice LW, Berkowitz RS, Goff BA. Pseudomyxoma peritonei: a review of 23 cases Obstet Gynecol 1994; 84(1): 17-21.

[30] Witkamp AJ, de Bree E, Kaag MM, van Slooten GW, van Coevorden F, Zoetmulder FA. Extensive surgical cytoreduction and intraoperative hyperthermic intraperitoneal chemotherapy in patients with pseudomyxoma peritonei. Br J Surg 2001; 88(3): 458-463. 
الورم المخاطي الكاذب في البريتون : التشخيص والعلاج

\section{خالد حسين سيت}

قسم أمراض النساء والتوليد ، كلية الطب ، جامعة الملك عبدالعزيز

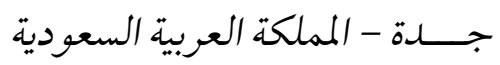

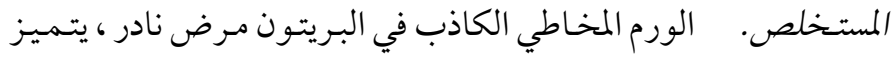

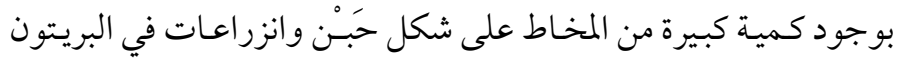

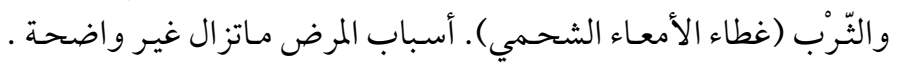

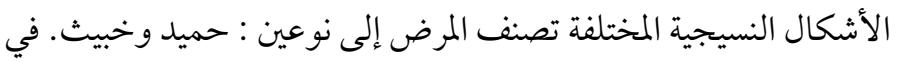

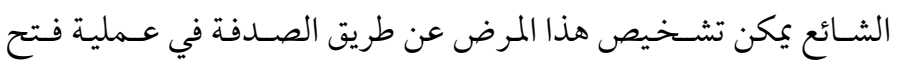

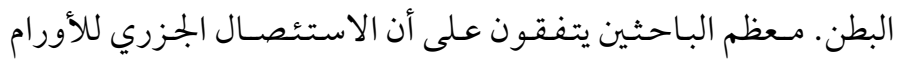

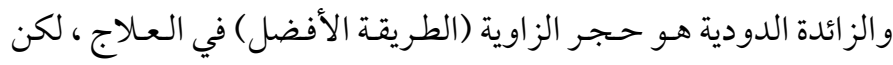

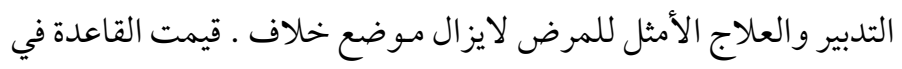

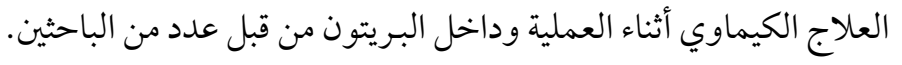

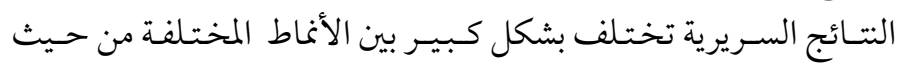

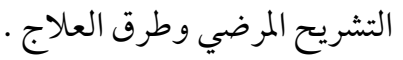

